\title{
X-ray emission from an FU Orionis star in early outburst: HBC 722
}

\author{
Armin Liebhart ${ }^{1}$, Manuel Güdel ${ }^{1}$, Stephen L. Skinner ${ }^{2}$, and Joel Green ${ }^{3}$ \\ 1 Department of Astrophysics, University of Vienna, Türkenschanzstrasse 17, 1180 Vienna, Austria \\ e-mail: [armin.liebhart; manuel.guedel]@univie.ac.at \\ 2 CASA, University of Colorado, Boulder, CO 80309-0389, USA \\ e-mail: stephen.skinner@colorado.edu \\ 3 Department of Astronomy, The University of Texas at Austin, 2515 Speedway, Stop C1400, Austin, TX 78712-1205, USA \\ e-mail: joel@astro.as.utexas.edu
}

Received 21 August 2014 / Accepted 17 September 2014

\section{ABSTRACT}

\begin{abstract}
Aims. We conducted the first X-ray observations of the newly erupting FU Ori-type outburst in HBC 722 (V2493 Cyg) with the aim to characterize its X-ray behavior and near-stellar environment during early outburst.

Methods. We used data from the XMM-Newton and Chandra X-ray observatories to measure X-ray source temperatures and luminosities as well as the gas column densities along the line of sight toward the source.

Results. We report a Chandra X-ray detection of HBC 722 with an X-ray luminosity of $L_{\mathrm{X}} \approx 4 \times 10^{30} \mathrm{ergs} \mathrm{s}^{-1}$. The gas column density exceeds values expected from optical extinction and standard gas-to-dust ratios. We conclude that dust-free gas masses are present around the star, such as strong winds launched from the inner disk, or massive accretion columns. A tentative detection obtained by $X M M$-Newton two years earlier after an initial optical peak revealed a fainter X-ray source with only weak absorption.
\end{abstract}

Key words. stars: individual: HBC 722 - stars: pre-main sequence $-\mathrm{X}$-rays: stars

\section{Introduction}

The small group of FU Ori stars (FUors) are pre-main sequence stars showing giant, long-lasting optical outbursts (Herbig 1977). The defining photometric feature of an FUor is a dramatic increase in optical brightness ( $\Delta V \gtrsim 4 \mathrm{mag}$ ), followed by a slow decline that can last from decades to centuries. This evolution is accompanied by a change from a typical T Tauri stellar spectrum to an $\mathrm{F}-\mathrm{G}$ supergiant spectrum in the optical and to a late-type ( $\mathrm{K}$ and later) giant spectrum in the near-infrared. Other important features are heavily blueshifted absorption lines (indicating velocities $>100 \mathrm{~km} \mathrm{~s}^{-1}$ ), CO overtone absorption bands around $2 \mu \mathrm{m}$, and almost no lines in emission, with the significant exception of $\mathrm{H} \alpha$. The few emission lines show P Cygni profiles, which indicates strong winds, again especially in $\mathrm{H} \alpha$. Furthermore, FU Ori stars in outburst are accompanied by newly formed reflection nebulae. The theory for FUor outbursts assumes a cataclysmic accretion event in which the accretion rate increases by a factor of 100 or more during a relatively short time interval ( $\$ 100$ years; e.g., Bell \& Lin 1994; Vorobyov \& Basu 2006).

A second group of eruptive variables, EXors (after their prototype EX Lup, Herbig 2008) exhibit similar observational features albeit with more modest amplitudes and on shorter time scales of several months to a few years. Optically, an EXor appears as a T Tauri star, while a FUor shows a much broader optical and near-IR peak that is dominated by the hot inner disk that outshines the central star.

$\mathrm{X}$-ray studies of FUors and EXors are interesting because strong accretion may change the magnetic topology in the stellar environment. The EXor V1647 Ori revealed a rapid increase in the X-ray flux by a factor of $\approx 30$ that closely tracked the optical and near-infrared light curves (Kastner et al. 2006). The $\mathrm{X}$-ray spectra hardened during the peak and softened during the decay. Extremely high temperatures of about $6 \times 10^{7} \mathrm{~K}$ indicated magnetic reconnection in star-disk magnetic fields. In contrast, V1118 Ori showed X-ray variations by no more than a factor of two during its 1-2 mag optical brightness increase on time scales of 50 days, but instead revealed a remarkable softening during the outburst, which indicates that the hot plasma disappeared during that episode (Audard et al. 2005).

$\mathrm{X}$-rays from FUors have been more elusive. Although two classical FUors have been recorded in X-rays so far, namely the prototype FU Ori (Skinner et al. 2006b) and V1735 Cyg (Skinner et al. 2009), these observations occurred long after the initial outbursts, during the gradually declining phase. Both revealed a high-temperature plasma $(k T>5 \mathrm{keV})$, which has been assumed to be the result of coronal activity. The $\mathrm{X}$-ray properties resemble those of Class I protostars. Two other FUors, V1057 Cyg and V1515 Cyg, remain X-ray nondetections (Skinner et al. 2009).

Here, we report the first X-ray detection of an FUor in the initial stages of its outburst. The recently erupting HBC 722 is the first and so far only FUor that has been monitored from its early outburst phase to the (presumably) main peak in all available wavelength bands (Dunham et al. 2012; Sung et al. 2013; Green et al. 2013; Semkov et al. 2014). We obtained three $\mathrm{X}$-ray observations during the initial rise of the optical light, during a subsequent minimum, and during the following second maximum. HBC 722 has been frequently studied in the optical and near-infrared and has meanwhile been classified as a genuine FUor (Semkov et al. 2012). The interest in its highenergy radiation was initially spurred by its apparent detection with Swift (Pooley \& Green 2010), although we caution that as demonstrated below - the field around our target is very complex and crowded in X-rays for the XMM-Newton and Swift angular resolution. There are no pre-outburst X-ray observations of HBC 722. 
A\&A 570, L11 (2014)

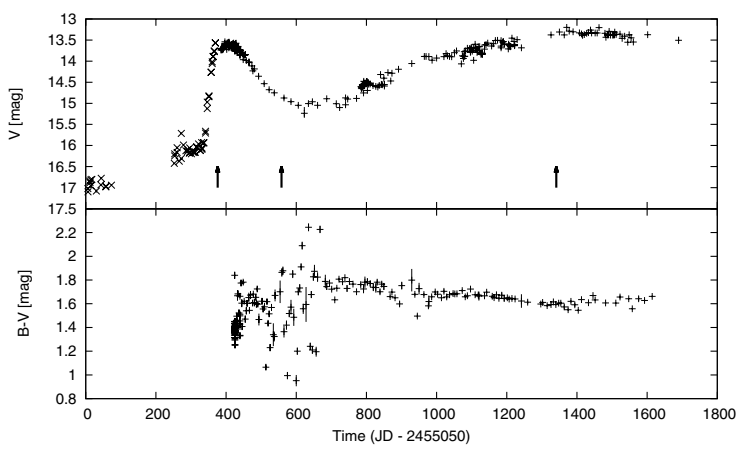

Fig. 1. $V$ (top) and $B-V$ (bottom) light curves of HBC 722 from aavso.org ( + symbols), complemented by $R$-band data from Miller et al. (2011) (top, early rise phase, $\times$ symbols). The three arrows mark the XMM1, XMM2, and CXO1 observing dates (left to right; Table 1).

\section{HBC 722}

The newly detected FUor HBC 722 (V2493 Cyg, LkH $\alpha 188$ G4) (Semkov et al. 2010) is located at a distance of 520 pc (Green et al. 2013) and is only the second FUor that has been observed prior to its outburst. Its pre-outburst characteristics (Cohen \& Kuhi 1979) indicate that it was an emission-line star in the spectral range K7-M0, that is most likely a classical $\mathrm{T}$ Tauri star (CTTS).

HBC 722 has become an object of great interest because its eruption was detected during its initial phase, and excellent photometric and spectroscopic pre-outburst data are available (Sect. 4). Its luminosity started to increase in May 2010 and reached a first maximum in October 2010 (Fig. 1), making it the fastest rise ever recorded for an FUor (Semkov et al. 2012). After a very fast initial decline until April 2011, it started to increase again and only recently reached a plateau. HBC 722 exhibits all defining features of a classical FUor. Its bolometric luminosity, $L_{\text {bol }}$, increased from $0.7 L_{\odot}$ to $12 L_{\odot}$ (Miller et al. 2011), which is an increase at the lower end for the class. The calculated accretion rate of $\mathrm{HBC} 722$ of $\sim 10^{-6} M_{\odot} \mathrm{yr}^{-1}$ also lies at the lower end of the class (Kóspál et al. 2011). But like other FUors, HBC 722 changed its spectrum from typical CTTS characteristics to a G3 supergiant in the optical and a K-type giant in the near- to midinfrared (Semkov et al. 2012). It only shows one detected emission line, namely $\mathrm{H} \alpha$ (Semkov \& Peneva 2011). The accompanying reflection nebula has grown to $\sim 2400 \mathrm{AU}$ in 2011 (Miller et al. 2011).

\section{Observations, data reduction, and analysis}

We requested XMM-Newton (Jansen et al. 2001) target of opportunity time twice to obtain an early X-ray view of HBC 722 (observations XMM1/2). Because of the complex source region with its many faint $\mathrm{X}$-ray sources, we additionally obtained Chandra X-ray Observatory (Weisskopf et al. 2000) guest observer time (observation CXO1). Table 1 summarizes dates, exposure times, number of counts in the source extraction area and net counts after background subtraction, and detection status of all observations; the X-ray observation times are marked in Fig. 1.

For the Chandra observation we used the Advanced CCD Imaging Spectrometer ACIS-S (Garmire et al. 2003) in vfaint mode. We reprocessed the level 2 event file using Chandra Interactive Analysis of Observations (CIAO) vers. 4.4, applying calibration data from CALDB version 4.4.8. Source detection was performed with the CIAO task wavdetect. We extracted the $0.5-10 \mathrm{keV}$ source spectrum of the clearly detected
Table 1. Log for HBC 722 X-ray observations.

\begin{tabular}{llllll}
\hline \hline $\begin{array}{l}\text { Obs. } \\
\text { code }\end{array}$ & ObsID & $\begin{array}{l}\text { Date } \\
(\mathrm{y} / \mathrm{m} / \mathrm{d})\end{array}$ & $\begin{array}{l}\text { Exp. } \\
\text { time }^{a}(\mathrm{ks})\end{array}$ & $\begin{array}{l}\text { Source } \\
\text { counts }^{b}\end{array}$ & Det. \\
\hline XMM1 & 656780701 & $2010 / 11 / 25$ & 16.85 & - & no \\
XMM2 & 656781201 & $2011 / 05 / 26$ & 17.56 & $19.3 \pm 6.1$ yes? \\
CXO1 & 14545 & $2013 / 07 / 17$ & 29.68 & $19.1 \pm 4.6$ & yes \\
\hline
\end{tabular}

Notes. ${ }^{(a)}$ Exp. time corresponds to LIVETIME; ${ }^{(b)}$ net source counts in 0.3-7 keV band for XMM2 (pn camera); $0.5-10 \mathrm{keV}$ for CXO1.

HBC 722 using a circular source area with radius $=1.76^{\prime \prime}$, and a background spectrum from a large source-free area on the same $\mathrm{CCD}$, using the CIAO specextract tool that also delivers the response matrix.

The XMM-Newton observations were obtained by the European Photon Imaging Cameras (EPIC). Given the faintness of the possible HBC 722 source, we report only $0.3-7 \mathrm{keV}$ results from the pn camera (Strüder et al. 2001), but the MOS cameras also show a marginal excess above background at the HBC 722 position. Data were reprocessed with the Scientific Analysis System (SAS, version 12.0.1 ${ }^{1}$ ). We performed source detection using the CIAO wavdetect task with a point spread function with a $40 \%$ encircled energy radius of $6^{\prime \prime 2}$. We extracted spectra for the source (within 4.5" around the bestmatching XMM2 source) and the background (from a large, source-free area) using the task evselect. The programs rmfgen and arfgen created the redistribution matrix (rmf) and ancillary response files (arf).

We modeled the observed spectra with XSPEC (Arnaud 1996), using a combination of a gas absorption column density (wabs model) and a spectrum of a collisionally ionized plasma (vapec model). The element abundances were adopted from the XEST project (Güdel et al. 2007), corresponding to typical values for pre-main sequence stars. Our model thus delivers the gas column density $N_{\mathrm{H}}$ along the line of sight toward the emitting source, the (average) source temperature, $T$, and a volume emission measure, EM. We derived the flux by integrating over the energy range $0.3-10 \mathrm{keV}$ and calculated the X-ray luminosity $L_{\mathrm{X}}$ using a stellar distance of $d=520 \mathrm{pc}$. We conducted a $2 \mathrm{D}$ parameter study by evaluating the best fits for any given combination of $N_{\mathrm{H}}$ and $k T$, and by determining the $90 \%$ confidence level for the two parameters of interest.

\section{Results}

The CIAO task wavdetect found a $7.9 \sigma$ X-ray source in $\mathrm{CXO} 1$ comprising 20 counts at $\mathrm{RA}(2000.0)=20 \mathrm{~h} 58 \mathrm{~m} 17.07 \mathrm{~s} \pm 0.01 \mathrm{~s}$, $\delta(2000.0)=43^{\circ} 53^{\prime} 43.21^{\prime \prime} \pm 0.05^{\prime \prime}$, offset by only $\approx 0.50^{\prime \prime}$ from the expected position, $\mathrm{RA}(2000.0)=20 \mathrm{~h} 58 \mathrm{~m} 17.025 \mathrm{~s} \pm$ $0.006 \mathrm{~s}, \delta(2000.0)=43^{\circ} 53^{\prime} 43.39^{\prime \prime} \pm 0.03^{\prime \prime}\left(\right.$ Cutri et al. 2003) ${ }^{3}$. The XMM-Newton observation XMM1 did not reveal any significant source at the expected position of HBC 722, but the region is, at the angular resolution of XMM-Newton, very crowded and potentially blurred by other point-like X-ray sources (Fig. 2). Wavdetect revealed a point-like $3 \sigma$ X-ray source in XMM2 at RA(2000.0) $=20 \mathrm{~h} 58 \mathrm{~m} 17.19 \mathrm{~s} \pm 0.038 \mathrm{~s}$, $\delta(2000.0)=43^{\circ} 53^{\prime} 41.31^{\prime \prime} \pm 0.36^{\prime \prime}$, offset from the expected position by $2.7^{\prime \prime}$, corresponding to $\approx 0.7 \sigma \mathrm{rms}$ uncertainty of the

1 See "User Guide to the XMM-Newton Science Analysis System", Issue 7.0, 2010 (ESA: XMM-Newton SOC).

2 See XMM-Newton Users Handbook Issue 2.1, Sect. 3.2.1.1.

3 The rms positional uncertainty for an on-axis point source is $\approx 0.42^{\prime \prime}$; see CXO Users Manual, http://asc . harvard. edu/proposer/POG 


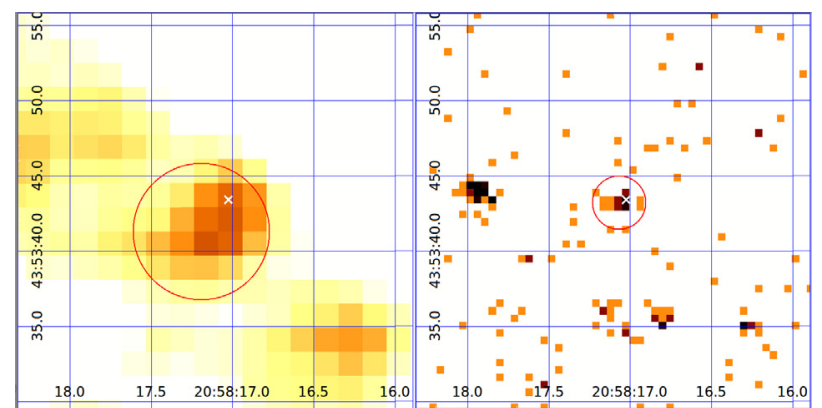

Fig. 2. Maps for XMM2 (left; pixel size 1.6", energy range $=0.3-7 \mathrm{keV}$ ) and CXO1 observations (right; pixel size 0.49", energy range $=0.5-10 \mathrm{keV}$ ). The circles show the extraction regions (radii $=4.5^{\prime \prime}$ and $1.76^{\prime \prime}$ for XMM2 and CXO1, respectively), centered at the wavdetect source coordinates. The crosses mark the 2MASS position (Cutri et al. 2003). The XMM-Newton image has been smoothed with a Gaussian.

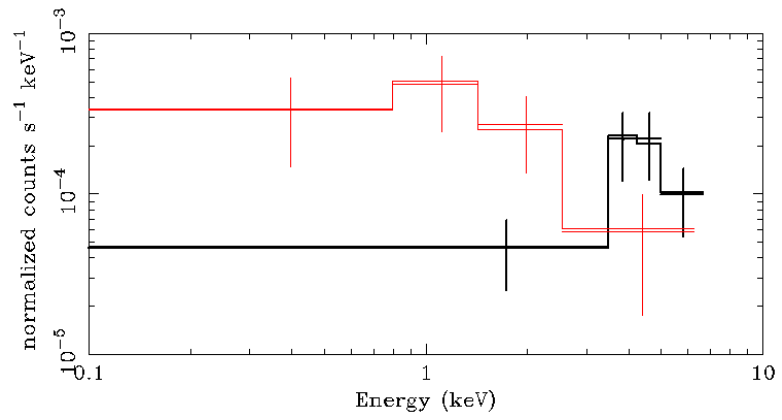

Fig. 3. Observed spectra (error bars) and fits (histograms) of CXO1 (black) and XMM2 (red), binned to a minimum of 5 and $7 \mathrm{cts} / \mathrm{bin}$, respectively.

absolute XMM-Newton-pointing accuracy ${ }^{4}$. Similar offsets were found for bright sources in the field. Because of some potential contamination by neighboring sources, we consider this faint detection tentative but useful in the context of the later Chandra detection.

Table 2 shows the results for the XMM 2 and CXO1 observations; $L_{\mathrm{X}}$ is the unabsorbed luminosity in the $0.3-10 \mathrm{keV}$ band. The XSPEC norm is defined as EM $/\left(4 \pi 10^{14} d^{2}\right), d$ being the distance to the star. The near-absence in the Chandra spectrum of $\mathrm{X}$-ray counts below $\approx 3 \mathrm{keV}$ and a spectral peak around $4-5 \mathrm{keV}$ (Fig. 3) require very high $N_{\mathrm{H}}$. Our best fit to a spectrum rebinned to at least five counts per bin delivered $N_{\mathrm{H}} \approx 1.4 \times 10^{23} \mathrm{~cm}^{-2}$ (90\% confidence range: $(4.4-56) \times 10^{22} \mathrm{~cm}^{-2}$ ). For a standard interstellar gas-to-dust ratio, we expect an optical extinction, $A_{V}$, of approximately $A_{V} \approx N_{\mathrm{H}} /\left(1.8 \times 10^{21} \mathrm{~cm}^{-2}\right) \approx 80 \mathrm{mag}$ or higher, in disagreement with optically determined $A_{V}$ measurements, which are sensitive primarily to dust absorption (see below). Given the few counts for the $\chi^{2}$ statistics, we alternatively used unbinned data in conjunction with the $\mathrm{C}$ statistic (Cash 1979, Table 2). We fully confirm the high $N_{\mathrm{H}}$ and found very similar $90 \%$ confidence ranges for all parameters as for the binned data.

To assess the observed $A_{V}$, we extracted $B-V$ colors from the online data of the American Association of Variable Star Observers (AAVSO; Henden 2014). $B-V \approx 1.7 \mathrm{mag}$ remains nearly constant during the recording time (Fig. 1 bottom). The G3 supergiant optical spectrum of HBC 722 (Semkov et al. 2012) corresponds to $(B-V)_{0} \approx 0.9 \mathrm{mag}$

\footnotetext{
4 The rms positional uncertainty for an on-axis point source is $\approx 4^{\prime \prime}$; see XMM-Newton Users Handbook, http://xmm.esac.esa.int/ external/xmm_user_support/documentation/uhb/index.html
}

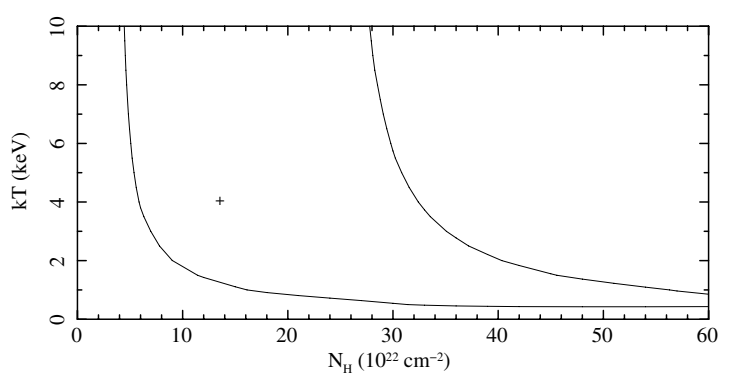

Fig. 4. 90\% confidence level range for $k T$ vs. $N_{\mathrm{H}}$ for the CXO1 observation using binned data. The cross marks the best fit.

Table 2. Results for the XSPEC spectral model fits.

\begin{tabular}{llll}
\hline \hline Parameter & XMM2 $(\mathrm{pn})$ & CXO1 (ACIS) & CXO1 unbin $^{a}$ \\
\hline$N_{\mathrm{H}} / 10^{22} \mathrm{~cm}^{-2}$ & $0.08(\ldots, 0.72)$ & $13.8(4.4,55.9)$ & $20.5(3.3,55.2)$ \\
$k T[\mathrm{keV}]$ & $8.4(1.3, \ldots)$ & $4.1(0.55, \ldots)$ & $2.3(0.55, \ldots)$ \\
norm $/ 10^{-5} \mathrm{~cm}^{-3}$ & $0.9(0.4,2.3)$ & $7.3(1.6,1840)$ & $20.4(1.6,1900)$ \\
$L_{\mathrm{X}, \text { best }}\left[\mathrm{erg} \mathrm{s}^{-1}\right]$ & $4.3 \times 10^{29}$ & $3.5 \times 10^{30}$ & $7.8 \times 10^{30}$ \\
Statistic/d.o.f. & $0.04 / 1$ & $0.04 / 1$ & $17.9 / 17$ \\
\hline
\end{tabular}

Notes. Norm $=\mathrm{EM} /\left(4 \pi 10^{14} d^{2}\right)$, where $d=$ distance to the $\operatorname{star}(520 \mathrm{pc})$ and $L_{X}$,best is the unabsorbed $L_{X}$ for the best fit. Statistic/dof is $\chi^{2}$ for Cols. 2 and 3, and $C$ for Col. 4, divided by the number of degrees of freedom; $90 \%$ confidence ranges (for one parameter of interest) are given in parentheses. Ellipsis dots indicate an unconstrained error range. ${ }^{(a)}$ For unbinned data.

(Binney \& Merrifield 1998). We then used $A_{V}=R_{V} \times E(B-V)$, where $E(B-V)=(B-V)-(B-V)_{0}$ is the color excess and $R_{V}$ is the total-to-selective extinction. Applying the standard value for $R_{V}=3.1$ (Schultz \& Wiemer 1975), we obtain $A_{V} \approx 2.5$. Using $E(B-V)=N_{\mathrm{H}} / 5.8 \times 10^{21} \mathrm{~cm}^{-2}$ (Binney \& Merrifield 1998) for a standard interstellar gas-to-dust mass ratio, we expect only $N_{\mathrm{H}} \approx 4.6 \times 10^{21} \mathrm{~cm}^{-2}$.

The XMM2 results were derived from a spectrum binned to at least seven counts per bin. While the highest source temperature is essentially unconfined, the presence of soft photons in the spectral range of $0.5-1 \mathrm{keV}$ requires $N_{\mathrm{H}}$ to be moderate; we find $N_{\mathrm{H}} \approx 8 \times 10^{20} \mathrm{~cm}^{-2}$, with $90 \%$ confidence upper limits around $N_{\mathrm{H}} \approx 7.2 \times 10^{21} \mathrm{~cm}^{-2}$. No acceptable joint fit could be found for the XMM2 and CXO1 observations, demonstrating that the two $N_{\mathrm{H}}$ ranges are mutually exclusive. For standard interstellar gas-to-dust ratios, we expect $A_{V}$ of about $1 \mathrm{mag}$ for the best fit, in agreement with $B-V$ observations and in stark contrast to the CXO1 results.

\section{Discussion and conclusions}

The anomalously high gas absorption $(\mathrm{CX} 01)$ in the presence of rather weak optical extinction is similar to an observation in a class of strongly accreting $\mathrm{T}$ Tauri stars that exhibit a combination of two X-ray spectra, a soft component from a cool $(\approx 2 \mathrm{MK})$, only weakly absorbed source and a very hard component from a hot, strongly absorbed $\left(N_{\mathrm{H}}>10^{22} \mathrm{~cm}^{-2}\right)$ source (two-absorber X-ray or TAX phenomenology, e.g., Güdel et al. 2008). The classical FUor FU Ori shares these characteristics (Skinner et al. 2006a, 2010). The soft source in DG Tau has been identified with an X-ray jet close to the star (Güdel et al. 2008), while for FU Ori a companion may at least partly explain it (Skinner et al. 2010). In all cases, however, the hard source, attributed to a magnetically confined plasma (e.g., a corona), requires much higher gas column densities than expected from visual extinction and a standard interstellar gas-to-dust mass 
ratio. The proposed models either involve dust-depleted accretion streams from the disk to the star or dust-depleted winds launched from the inner disk.

To assess the plausibility of these models, we first estimated $N_{\mathrm{H}}$ from accretion streams using mass conservation for a stationary flow approximated to be isotropic and radial,

$n_{\mathrm{H}}=\frac{\dot{M}}{4 \pi r^{2} \mu m_{\mathrm{p}} v}$,

where $m_{\mathrm{p}} \approx 1.7 \times 10^{-24} \mathrm{~g}$ and $\mu \approx 1.3$ are the proton mass and the mean weight per particle for atomic gas. The mass accretion rate for HBC 722 is $\dot{M}=10^{-6} M_{\odot} \mathrm{yr}^{-1}$ (Kóspál et al. 2011). Halfway between disk border and stellar surface, the accretion stream velocity will have reached a value of about half the freefall velocity at the stellar surface for a mass element falling from the inner-disk border. Using the inner disk radius $r=2 R_{*}, R_{*} \approx$ $2 R_{\odot}$, and a stellar mass of $M_{*}=0.5 M_{\odot}$ (Green et al. 2013) we obtain $v \approx 126 \mathrm{~km} \mathrm{~s}^{-1}$ and therefore $n_{\mathrm{H}} \approx 4 \times 10^{12} \mathrm{~cm}^{-3}$ at $r \approx$ $1.5 R_{*}$. Integrating over one $R_{*}$ (from $2 R_{*}$ to the stellar surface) leads to $N_{\mathrm{H}}=5.6 \times 10^{23} \mathrm{~cm}^{-2}$, within the $90 \%$ confidence range of CXO1 results (Table 2).

We now consider winds launching from the innermost part of the disk. Considering the strong heating of the inner disk during an FUor outburst, we may expect winds, as indeed observed in FUors (Herbig et al. 2003) and in particular also for HBC 722 where they reach velocities of $500 \mathrm{~km} \mathrm{~s}^{-1}$ (Semkov et al. 2012). The gas of the inner disk is essentially dust-free because it is far inside the dust sublimation radius even for a normal CTTS; in any case, the disk temperature corresponding to a $\mathrm{G}$ supergiant spectrum exceeds the dust sublimation temperature. Optical extinction (due to dust) will therefore not be enhanced, while X-rays will still be absorbed by gas.

If such a dust-poor wind is launched from the inner disk region and expands approximately isotropically, then Eq. (1) analogously applies, where $\dot{M}$ now stands for the wind mass-loss rate. Integration of $n_{\mathrm{H}}$ along the line of sight through the wind from infinity to the disk border $\left(2 R_{*}\right)$ provides an estimate for $N_{\mathrm{H}}$ if we assume that the wind velocity is constant:

$N_{\mathrm{H}}=\int_{2 R_{*}}^{\infty} \frac{\dot{M}}{4 \pi r^{2} \mu m_{\mathrm{p}} v} \mathrm{~d} r=\frac{\dot{M}}{8 \pi \mu m_{\mathrm{p}} v R_{*}}$.

Using $R_{*} \approx 2 R_{\odot}$ (Green et al. 2013), $v \approx 500 \mathrm{~km} \mathrm{~s}^{-1}$ (Semkov et al. 2012), and $\dot{M} \approx 10^{-7} M_{\odot} \mathrm{yr}^{-1}$ (assuming $10 \%$ of the mass accretion rate as for CTTS, Hartigan et al. 1995), we find $N_{\mathrm{H}} \approx$ $1.7 \times 10^{22} \mathrm{~cm}^{2}$, somewhat lower than acceptable for the CXO1 observations, but our model assumptions are fairly crude.

The XMM1/2 observations do not fit into this picture. At face value, it seems that strong winds or accretion stream absorption did not prevail in XMM2, while XMM1 suffered from too much absorption, or the X-ray source was significantly dimmer. We suggest the following scenario:

The first optical peak was produced by an initial strong disk instability that rapidly led to enhanced accretion and possibly winds that attenuated all X-rays (XMM1). The initial outburst then ceased, leading into a more quiet phase during which the star was detected in X-rays (XMM2). Subsequently, a gradual increase to a lasting disk instability develops winds and accretion flows and triggers enhanced X-ray emission. Enhanced X-ray absorption is now evident (CXO1).

Direct support for XMM2 picking up a normal CTTS comes from the measured $L_{X}$. Telleschi et al. (2007) reported statistical correlations between $L_{\mathrm{X}}$ and stellar $L_{\mathrm{bol}}$ or mass for a large sample of CTTS in Taurus. Using $L_{\mathrm{bol}}=0.7 L_{\odot}$ and $M_{*}=0.5 M_{\odot}$, these best-fit relations (based on two different statistical regression methods) all lead to $L_{\mathrm{X}} \approx(4.2-5.0) \times 10^{29} \mathrm{erg} \mathrm{s}^{-1}$, which agrees well with our XMM2 observation.

Why $L_{X}$ increased by about an order of magnitude during the outburst peak is less clear (the increase seems to agree with estimates for classical FUors; Skinner et al. 2009, 2010). Potential candidates are reconnection events in dynamo-induced magnetic fields forming as a consequence of convection in the strongly heated, unstable disk; the absorbing medium in this case would be a wind. Alternatively, enhanced magnetic reconnection in magnetospheric star-disk fields subject to increased disturbance by the close-in disk would lead to hard emission, while the overlying accretion streams and winds would partially attenuate the X-rays. Future X-ray monitoring may help to clarify the situation.

Acknowledgements. We thank an anonymous referee for helpful comments. We acknowledge with thanks the variable star observations from the AAVSO International Database contributed by observers worldwide and used in this research. We thank the Project Scientist of XMM-Newton, Norbert Schartel, for approving our XMM-Newton Target of Opportunity request. J.G. and S.S. acknowledge support from Chandra award GO3-14012. The CXC is operated by the Smithsonian Astrophysical Observatory for and on behalf of the NASA under contract NAS8-03060. This publication is supported by the Austrian Science Fund (FWF).

\section{References}

Arnaud, K. A. 1996, in Astronomical Data Analysis Software and Systems V, eds. G. H. Jacoby \& J. Barnes, ASP Conf. Ser., 101, 17

Audard, M., Güdel, M., Skinner, S. L., et al. 2005, ApJ, 635, L81

Bell, K. R., \& Lin, D. N. C. 1994, ApJ, 427, 987

Binney, J., \& Merrifield, M. 1998, Galactic Astronomy (New Jersey: Princeton University Press)

Cash, W. 1979, ApJ, 228, 939

Cohen, M., \& Kuhi, L. V. 1979, ApJS, 41, 743

Cutri, R. M., Skrutskie, M. F., van Dyk, S., et al. 2003, VizieR Online Data Catalog: II/246

Dunham, M. M., Arce, H. G., Bourke, T. L., et al. 2012, ApJ, 755, 157

Garmire, G. P., Bautz, M. W., Ford, P. G., Nousek, J. A., \& Ricker, Jr., G. R. 2003, in X-Ray and Gamma-Ray Telescopes and Instruments for Astronomy, eds. J. E. Truemper, \& H. D. Tananbaum, SPIE Conf. Ser., 4851, 28

Green, J. D., Robertson, P., Baek, G., et al. 2013, ApJ, 764, 22

Güdel, M., Briggs, K. R., Arzner, K., et al. 2007, A\&A, 468, 353

Güdel, M., Skinner, S. L., Audard, M., Briggs, K. R., \& Cabrit, S. 2008, A\&A, 478, 797

Hartigan, P., Edwards, S., \& Ghandour, L. 1995, ApJ, 452, 736

Henden, A. A. 2014, Observations from the AAVSO International Database, http: //www . aavso.org

Herbig, G. H. 1977, ApJ, 217, 693

Herbig, G. H. 2008, AJ, 135, 637

Herbig, G. H., Petrov, P. P., \& Duemmler, R. 2003, ApJ, 595, 384

Jansen, F., Lumb, D., Altieri, B., et al. 2001, A\&A, 365, L1

Kastner, J. H., Richmond, M., Grosso, N., et al. 2006, ApJ, 648, L43

Kóspál, Á., Ábrahám, P., Acosta-Pulido, J. A., et al. 2011, A\&A, 527, A133

Miller, A. A., Hillenbrand, L. A., Covey, K. R., et al. 2011, ApJ, 730, 80

Pooley, D., \& Green, J. 2010, ATel, 3040, 1

Schultz, G. V., \& Wiemer, W. 1975, A\&A, 43, 133

Semkov, E., \& Peneva, S. 2011, Blg AJ, 17, 88

Semkov, E. H., Peneva, S. P., Munari, U., Milani, A., \& Valisa, P. 2010, A\&A, 523, L3

Semkov, E. H., Peneva, S. P., Munari, U., et al. 2012, A\&A, 542, A43

Semkov, E. H., Peneva, S. P., Ibryamov, S. I., \& Dimitrov, D. P. 2014, Blg AJ, 20, 59

Skinner, S., Güdel, M., Briggs, K., Melnikov, S., \& Audard, M. 2006a, Ap\&SS, 304,165

Skinner, S. L., Briggs, K. R., \& Güdel, M. 2006b, ApJ, 643, 995

Skinner, S. L., Sokal, K. R., Güdel, M., \& Briggs, K. R. 2009, ApJ, 696, 766

Skinner, S. L., Güdel, M., Briggs, K. R., \& Lamzin, S. A. 2010, ApJ, 722, 1654

Strüder, L., Briel, U., Dennerl, K., et al. 2001, A\&A, 365, L18

Sung, H.-I., Park, W.-K., Yang, Y., et al. 2013, J. Korean Astron. Soc., 46, 253

Vorobyov, E. I., \& Basu, S. 2006, ApJ, 650, 956

Weisskopf, M. C., Tananbaum, H. D., Van Speybroeck, L. P., \& O’Dell, S. L. 2000, in X-Ray Optics, Instruments, and Missions III, eds. J. E. Truemper, \& B. Aschenbach, SPIE Conf. Ser., 4012, 2 